\title{
Proposal for requirements on industrial AI solutions
}

\author{
Martin W Hoffmann ${ }^{1}$, Rainer Drath ${ }^{2}$ and Christopher Ganz ${ }^{3}$ \\ ${ }^{1}$ ABB AG, Corporate Research, 68526 Ladenburg, Germany \\ ${ }^{2}$ Hochschule Pforzheim, 75175 Pforzheim, Germany \\ ${ }^{3}$ ABB Ltd., 8050 Zürich, Switzerland \\ martin.w.hoffmann@de.abb.com
}

\begin{abstract}
The rise of artificial intelligence (AI) promises productivity gains in industrial practice. While IT technology offers a variety of technological advances, plant owners strive for stability and robustness of the production process. To overcome this tension field, we propose a set of 16 requirements for the development of industrial AI solutions to foster i) the adaptation process, ii) support the solution engineering and iii) ease the embedding into the existing system landscape while respecting iv) safety aspects to build up v) trust into industrial AI solutions. The proposed requirements can guide industrial stakeholders to focus on the right solution approach for specific production challenges and support them in voicing their own needs towards novel AI solutions. This will help AI developers to speed up time-to-market as well as to increase market acceptance of industrial AI solutions. Overall, specifying requirements on industrial AI will foster the acceptance and utilization rates of AI solutions in industrial practice.
\end{abstract}

Keywords: Artificial intelligence, AI, industrial AI, industrial production, requirements, digital twin, autonomy, manufacturing, Industrie 4.0, Industry 4.0, use-case.

\section{Introduction}

The introduction of software methods into industrial automation is currently a key source of innovation. E.g., the announced fourth industrial revolution (Industrie 4.0) aims for the introduction of internet technology into production and promises productivity gains across all phases of an industrial plant [1]. While IT technology promises a variety of possibilities or opportunities, plant owners aim for stability and robustness of the plant, requiring reliability and proven technology. This is a field of tension between feasibility and stability and related industrial requirements have been formulated [2].

The same now applies with the introduction of artificial intelligence (AI) into production. Not everything that is possible with mainstream AI is applicable in industry. Therefore, the authors propose a set of industrial requirements for AI solutions as a basic guideline for industrial AI developers and vendors. 


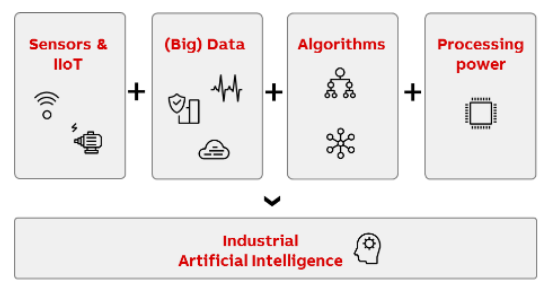

a)

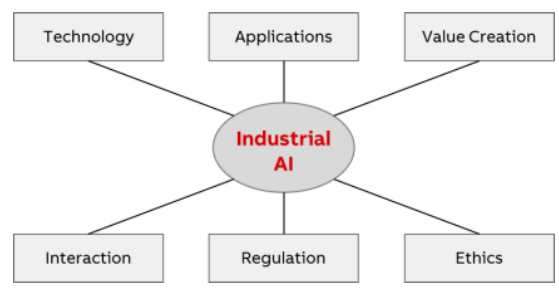

b)

Fig. 1. a) Ingredients comprising Industrial AI. b) Topics of discussion around industrial AI.

\subsection{Usage of AI in Industrial Production}

The application of AI technologies in industrial is not a new topic and has been subject to scientific investigations also during previous periods of AI research, e.g. [3,4]. More recently, research focused on the application of AI techniques in fault diagnosis and predictive maintenance [5-12] as well as decision support systems [5,13]. Current academic investigations aim at the coordination of the introduction of AI into all layers of production systems [14] as well as production-wide maintenance processes [15].

\subsection{Industrial AI}

Besides existing definitions of Industrial AI [16,17], we define Industrial Artificial Intelligence for this study as the combination of sensors \& IIoT, (big) data, algorithms and processing power (Fig. 1a). However, the discussions about Industrial AI, as the authors experience them in daily practice, are not limited to technological aspects of AI, but rather mix questions about technology, industrial applications, value creation, human-AI-interaction, regulatory aspect as well as ethics (Fig. 1b). These broader understanding of Industrial AI bring up additional requirements from an industrial perspective, which need to be addressed for Industrial AI to unfold its full (positive) impact on an industrial production.

\section{Requirements on industrial AI}

The presented requirements were collected during the years 2017 and 2018 in an unstructured fashion during several industrial research projects and end-customer discussions in the field of AI-enabled production systems. The collected requirements can be sorted in five categories (Tab 1), which build upon another (Fig. 2). 


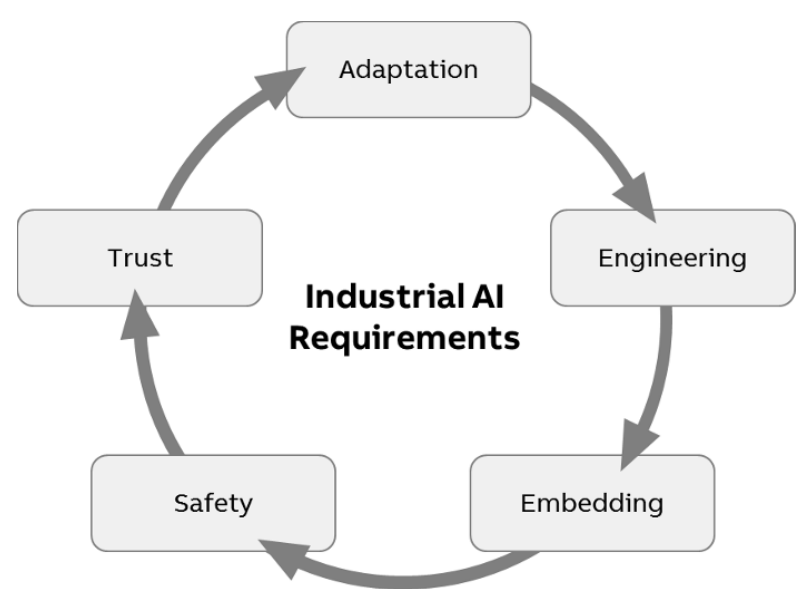

Fig. 2. Interdependence of requirements on Industrial AI.

\subsection{Adaption of Industrial AI systems}

The introduction of novel technology into production environments brings some specific requirements with it, as we deal mostly with existing production facilities, socalled brownfield environments. Brownfield environments are characterized by a zoo of existing IT and OT systems, as well as experienced employees running the production.

1) Stepwise introduction. Industrial AI should be introduced step by step so that it can initially validate itself in the context of production. A parallel operation with conventional automation technologies is desirable in terms of confidence generation. The power of decision should be gradually transferred from the conventional automation technology to the industrial AI, similar to the gradual increase of autonomy of the system [18]. It is to be noted though that for safety and reliability reasons, some critical functionality may remain in a conventional automation system. The Industrial AI system will thus be operating under the supervision of the safety governing system.

2) Human in the loop / system autonomy. During the introduction phase of Industrial AI in production systems, human should remain in control of all decisions, i.e. the Industrial AI may only serve as expert system or decision support system, corresponding to lower levels of autonomy [18]. Only by time, the autonomy of the Industrial AI may increase.

3) Data availability. AI systems usually require massive amounts of data. In industrial settings we usually struggle far more with the data quality [15], including the amount of relevant information contained in large amounts of machine data [19-21]. Furthermore, data from heterogeneous OT and IT systems needs to be accessed, usually 
bringing legal, contractual, cultural, commercial, technical and security topics on the table [21].

Table 1. Overview of proposed requirements on Industrial AI solutions.

\begin{tabular}{|c|c|}
\hline Area & Requirement \\
\hline Adaption & $\begin{array}{l}\text { 1. Stepwise introduction } \\
\text { 2. Human in the loop } \\
\text { 3. Data availability }\end{array}$ \\
\hline Engineering & $\begin{array}{l}\text { 4. Virtual learning } \\
\text { 5. Adaptation } \\
\text { 6. Simplicity (hiding of complexity) }\end{array}$ \\
\hline Embedding & $\begin{array}{l}\text { 7. Stacking of AI decisions } \\
\text { 8. Trust space and borders } \\
\text { 9. Knowledge distribution }\end{array}$ \\
\hline Safety / Security & $\begin{array}{l}\text { 10. Safety } \\
\text { 11. Robust against adversarial inputs }\end{array}$ \\
\hline Trust & $\begin{array}{l}\text { 12. Traceability and transparency of decisions } \\
\text { 13. Bias-free } \\
\text { 14. Confidence measure } \\
\text { 15. Trust / quality classification } \\
\text { 16. Proof of capabilities }\end{array}$ \\
\hline
\end{tabular}

\subsection{Engineering of Industrial AI systems}

Once general problems with the introduction of Industrial AI systems are overcome, the engineering of such novel systems requires special attention.

4) Simplicity / hiding of complexity. Industrial AI solutions may not be designed a way such that the adaptation to new production lines or customer settings requires eminent engineering efforts, as this would counter a main advantage of AI-based solutions. The user of AI-based production systems will most-likely not have a mathematical or computer science education and thus also the usage and operation of industrial AI systems shall be simple and hide all otherwise required technical complexity. A more simplistic design of Industrial AI solution will also lead to greater robustness of the system [23]. 
5) Virtual learning. Industrial AI should learn over time and the learning should not be limited to physical tests but should additionally be executed in virtual environments as much as possible, e.g. utilizing digital twin concepts [24-26]. In the result, e.g. a robot can optimize its path virtually.

6) Adaptation. Industrial AI should continuously adapt its capabilities to a changing production environment. Environmental changes (e.g. position drift) need to be detected and handled correctly.

\subsection{Embedding of Industrial AI system in existing production system landscape}

Industrial AI systems independent of their embedding in either greenfield or brownfield environments will interact with several other OT and IT systems in the production facility and beyond. Besides well-known technical requirements of the interfaces between such systems, further requirements will come up in light of the broader capabilities of Industrial AI systems.

7) Trust space and trust borders. In case that industrial AI provides functionality, which cannot be achieved with traditional automation technology (e.g. gripping of loose parts in box) and hence no classic system backup is possible, a dedicated checkpoint is required which allows a human to prove the plausibility of taken decisions.

8) Knowledge distribution. Industrial AI should be able to distribute its knowledge and learnings to other industrial AIs, either directly or via superior systems or communication networks.

9) Stacking of AI decisions. Industrial AI should initially not base its conclusions on data that have themselves been created by another AI.

\subsection{Safety and Security of Industrial AI systems}

Safety and security are both in the OT and IT world hard requirements, although failures in production systems such as chemical processes or power plants can cause far bigger damage than compromised mainstream AI applications. The individual requirements regarding safety and security may fill complete studies by their own. At this, we would like to only highlight two requirements exemplarily.

10) Safety. Vendors and providers of industrial AI-enabled machines or production systems need to ensure that the they work safe according to the EU Machinery Directive 2006/42/EC [27] as well as categorized according to IEC61508 Safety Integrity Levels (SIL), i.e. the industrial AI may not pose a danger to humans in any possible operation 
condition and the risk of failure should be made transparent according to the IEC reaching a best possible SIL. Industrial AI solution should in the future also be able to prove that they are safe even under recursive self-improvement [28].

11) Robustness against adversarial inputs. Industrial AI needs to be robust against accidental and intended adversarial inputs to ensure a maximum of protection of the production process.

\subsection{Trust in functionality of Industrial AI systems}

Production workers and managers fully trust today's production systems. This trust needs to be carried over into AI-enabled production systems, especially in the light of prominent examples of failing mainstream AI [29].

12) Traceability and transparency of decisions. Industrial AI should be able to explain its decisions, e.g. by means of visualizations. Errors in the industrial AI's assumptions shall be recognizable and correctable. Example: if a workpiece is recognized by industrial AI, the considered elements of the workpiece should be visualized. False assumptions should be highlighted and correctable.

13) Trust/quality classification. Industrial AI should be divided into trust/quality classes, which are backed by statistics. This is to express the probability of failure of the AI, e.g. determined by experiments or field tests. Industrial AI can be stacked if the underlying AI fulfills a sufficient trust category.

14) Proof of capability. Industrial AI should allow to check its capabilities and limitations in a determined and safe space, e.g. in a virtual environment or a test run.

15) Bias-free. Industrial AI needs to be constructed free of bias, e.g. treating equipment of all vendors in the same manner. Besides still feeling superior to machines in the consumer world when looking at failing AI [29], Industrial AI may never suffer from bias in data leading to a negative impact on production. As such Industrial AI systems need to be thoroughly tested before the usage in a productive environment, e.g. in the virtual layer of a CPS.

16) Confidence measure. Expectation on Industrial AI system higher than on industrial worker or consumer AI solution. Especially in European countries Industrial AI system are expected to have $100 \%$ solution rate and $0 \%$ error rate. This of course cannot be achieved, neither by a technical system nor by a human expert. It is therefore required that Industrial AI system provide a confidence measure together with decision making or action recommendations. 


\section{Discussion}

The aim of the presented study is to trigger further discussions about requirements on the introduction of AI into industrial production environments. The presented list of requirements may not be conclusive but covers many aspects specific to Industrial AI solutions. Further requirements left out in the presented list may cover topics such as data / analytics privacy [30], ethical implication [31], potential malicious use of AI [32] as well as AI-vendor lock-in [23].

The term of "Industrial AI" has previously been defined differently to the definition presented in the introduction of this paper $[16,17]$. Our definition is however not contradicting the previous definitions, but rather sharpens the understanding of Industrial AI in the context of the present study. Previously, the definition of Industrial AI also included application requirements [17] or Industrial AI was thought to "function as a bridge connecting academic research outcomes in AI to industry practitioners" [16], which both fit to the topics surrounding Industrial AI (Fig 1b), from our perspective.

It is interesting to note that some of the presented requirements correspond with identified requirements on Industrial AI correspond to research fields recently identified for Scientific Machine Learning [33]. We foresee future developments of Industrial $\mathrm{AI}$ in the areas of i) production system autonomy [18], ii) product life cycle management [15], especially because AI life cycles will become more complex to manage [23], iii) virtual industrial assistants [34], iv) explainable AI [35] and v) the seamless fusion of different data pools in production sites.

\section{Conclusion}

The introduction of Industrial AI brings up a tension field between plant owners striving for reliability and stability of the production and AI technologies entering the production systems. We proposed a set of 16 requirements in five categories as guidelines for Industrial AI developers as well as Industrial AI users to foster the adaptation of the new opportunities arising from AI in the industrial domain.

\section{Acknowledgements}

The work has been supported by the EU ECSEL project Productive 4.0.

\section{References}

1. Krueger, MW, et al. "A new era: ABB is working with the leading industry initiatives to help usher in a new industrial revolution." ABB Review 4/2014: 70-75, 2014.

2. Drath, R and Horch, A. "Industrie 4.0 - hit or hype?", IEEE Industrial Electronics Magazine 01/2014; 8(2):56-58, 2014.

3. Fox, M. S. "Industrial applications of artificial intelligence." Robotics 2.4: 301-311, 1986. 
4. Parunak, H. "Applications of distributed artificial intelligence in industry." Foundations of distributed artificial intelligence 2, 1996.

5. Yam, RCM, et al. "Intelligent predictive decision support system for condition-based maintenance." The International Journal of Advanced Manufacturing Technology 17.5: 383391, 2001.

6. Li, Z, Wang, Y and Wang, K-S. "Intelligent predictive maintenance for fault diagnosis and prognosis in machine centers: Industry 4.0 scenario." Advances in Manufacturing 5.4: 377 $387,2017$.

7. Sarda-Espinosa, A, Subbiah, S and Bartz-Beielstein, T. "Conditional inference trees for knowledge extraction from motor health condition data." Engineering Applications of Artificial Intelligence 62: 26-37, 2017.

8. Rødseth, H, Schjølberg, P and Marhaug, A. "Deep digital maintenance." Advances in Manufacturing 5.4: 299-310, 2017.

9. Ottewill, JR., and Orkisz, M. "Condition monitoring of gearboxes using synchronously averaged electric motor signals." Mechanical Systems and Signal Processing 38.2: 482-498, 2013.

10. Cecílio, IM., et al. "Nearest neighbors method for detecting transient disturbances in process and electromechanical systems." Journal of Process Control 24.9: 1382-1393, 2014.

11. Amihai, I, et al. "Modeling Machine Health Using Gated Recurrent Units with Entity Embeddings and K-Means Clustering." 2018 IEEE 16th International Conference on Industrial Informatics (INDIN), 2018.

12. Amihai, I, et al. "An Industrial Case Study Using Vibration Data and Machine Learning to Predict Asset Health." IEEE 20th Conference on Business Informatics (CBI). Vol. 1, 2018.

13. Atzmueller, M, et al. "Big data analytics for proactive industrial decision support." atp magazin 58.09: 62-74, 2016.

14. Wan, J, et al. "Artificial intelligence for cloud-assisted smart factory." IEEE Access 6: 55419-55430, 2018.

15. Zhang, Y, et al. "A big data analytics architecture for cleaner manufacturing and maintenance processes of complex products." Journal of Cleaner Production 142: 626-641, 2017.

16. Lee, J, et al. "Industrial Artificial Intelligence for industry 4.0-based manufacturing systems." Manufacturing letters 18: 20-23, 2018.

17. Zhang, , et al. "A reference framework and overall planning of industrial artificial intelligence (I-AI) for new application scenarios." The International Journal of Advanced Manufacturing Technology: 1-23, 2018.

18. Gamer, T. et al., "The Autonomous Industrial Plant - Future of Process Engineering, Operations and Maintenance," in 12th International Conference on Dynamics and Control of Process Systems (DYCOPS), 2019.

19. Gitzel, G, Turrin, S and Maczey, S. "A data quality dashboard for reliability data." 2015 IEEE 17th Conference on Business Informatics. Vol. 1. IEEE, 2015.

20. Gitzel, R. "Data Quality in Time Series Data: An Experience Report." CBI (Industrial Track). 2016.

21. Gitzel, R, Subbiah, S and Ganz, C. "A Data Quality Dashboard for CMMS Data." ICORES. 2018.

22. Deloitte LLP, "Realising the economic potential of machine-generated, nonpersonal data in the EU", available at https://www.vodafone.com/content/dam/vodafone-images/public-policy/reports/pdf/Realising_the_potential_of_IoT_data_report_for_Vodafone.pdf, last accessed 24.04.2019, 2018.

23. Dutta, D, et al. "Towards \#consistentAI." First International Conference on Artificial Intelligence for Industries (AI4I), 2018. 
24. Tao, F, et al. "Digital twin-driven product design, manufacturing and service with big data." The International Journal of Advanced Manufacturing Technology 94.9-12: 3563-3576, 2018.

25. Wagner, $\mathrm{C}$, et al. "The role of the Industry 4.0 asset administration shell and the digital twin during the life cycle of a plant." 22nd IEEE International Conference on Emerging Technologies and Factory Automation (ETFA), 2017.

26. Malakuti, S, and Grüner, S. "Architectural aspects of digital twins in IIoT systems." Proceedings of the 12th European Conference on Software Architecture: Companion Proceedings, 2018.

27. European Commission, "Directive 2006/42/EC of the European Parliament and of the Council of 17 May 2006 on machinery, and amending Directive 95/16/EC (recast)", Official Journal of the European Union, L 157/24, 2016.

28. Yampolskiy, RV. "Artificial intelligence safety engineering: Why machine ethics is a wrong approach." Philosophy and theory of artificial intelligence. Springer, Berlin, Heidelberg. 389-396, 2013.

29. Yampolskiy, RV and Spellchecker, MS. "Artificial intelligence safety and cybersecurity: A timeline of AI failures." arXiv preprint arXiv:1610.07997, 2016.

30. Marrella, A et al. "Privacy-Preserving Outsourcing of Pattern Mining of Event-Log Data-A Use-Case from Process Industry." IEEE International Conference on Cloud Computing Technology and Science (CloudCom), 2016.

31. EC High Level Expert Group on Artificial Intelligence, "Ethics Guidelines for trustworthy AI", https://ec.europa.eu/digital-single-market/en/news/ethics-guidelines-trustworthy-ai (accessed 03.06.2019), 2019.

32. Brundage, M, et al. "The malicious use of artificial intelligence: Forecasting, prevention, and mitigation." arXiv preprint arXiv:1802.07228, 2018.

33. Baker, N, et al.. "Workshop Report on Basic Research Needs for Scientific Machine Learning: Core Technologies for Artificial Intelligence." United States: N. p., 2019. Web. doi:10.2172/1478744, 2019.

34. Schmidt, B, et al. "Industrial Virtual Assistants: Challenges and Opportunities." Proceedings of the 2018 ACM International Joint Conference and 2018 International Symposium on Pervasive and Ubiquitous Computing and Wearable Computers, 2018.

35. Gunning, D. "Explainable artificial intelligence (xai)." Defense Advanced Research Projects Agency (DARPA), nd Web, 2017. 


\section{Hoffmann et al.}

Open Access This chapter is licensed under the terms of the Creative Commons Attribution 4.0 International License (http://creativecommons.org/licenses/by/4.0/), which permits use, sharing, adaptation, distribution and reproduction in any medium or format, as long as you give appropriate credit to the original author(s) and the source, provide a link to the Creative Commons license and indicate if changes were made.

The images or other third party material in this chapter are included in the chapter's Creative Commons license, unless indicated otherwise in a credit line to the material. If material is not included in the chapter's Creative Commons license and your intended use is not permitted by statutory regulation or exceeds the permitted use, you will need to obtain permission directly from the copyright holder.

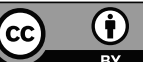

